\title{
Mean Muscle Attenuation within Paraspinal Muscle as a Determinant of Survival in Patients with Gastric Cancer Undergoing Gastrectomy
}

\section{Soomin An}

Hallym Polytechnic University

Wankyu Eo ( $\nabla$ wkeo@khu.ac.kr)

Kyung Hee University Hospital at Gangdong

YounJung Kim

Kyung Hee University

\section{Research Article}

Keywords: Gastrectomy, Stomach Neoplasms, Paraspinal Muscles, Muscle, Skeletal

Posted Date: January 13th, 2021

DOI: https://doi.org/10.21203/rs.3.rs-138661/v1

License: (c) (i) This work is licensed under a Creative Commons Attribution 4.0 International License.

Read Full License 


\section{Abstract}

Background: This study aimed to evaluate the prognostic potential of skeletal muscle mass and muscle quality at the level of the third lumbar vertebra (L3) using computerized tomography (CT) images in patients with stage I-III gastric cancer (GC) who underwent curative gastric resection.

Methods: Patients with stage I-III GC who underwent curative gastric resection between October 2006 and June 2014 were enrolled in this study. Demographic and clinical parameters were collected and analyzed. The muscle-related parameters (MRPs), such as the area of total abdominal wall musculature (SMA), area of paraspinal muscle (PMA), mean muscle attenuation (MA) within the total abdominal wall musculature (SMMA), and MA in the paraspinal muscle (PMMA), were measured at the L3 level using CT images. The Kaplan-Meier curve analyses and Cox proportional hazards model were applied to the MRPs, demographic, and clinical parameters to explore the overall survival (OS) and disease-free survival (DFS).

Results: Overall, data from 339 patients (233 men and 116 women) were analyzed in this study. In the multivariate Cox model, the elderly (hazard ratio $[\mathrm{HR}] 2.08,95 \%$ confidence interval $[\mathrm{Cl}] 1.30-3.32$, $\mathrm{p}=0.002)$, total gastrectomy (HR 2.14, 95\% Cl 1.32-3.48, $\mathrm{p}=0.002)$, stage (HR 3.41,95\% Cl 2.10-5.54, $\mathrm{p}<0.001)$, perineural invasion (HR 2.01, 95\% $\mathrm{Cl} 1.02-3.93, \mathrm{p}=0.042)$, prognostic nutritional index (PNI) (HR $0.91,95 \% \mathrm{Cl} 0.88-0.95, \mathrm{p}<0.001$ ), and PMMA (HR 2.36, 95\% Cl 1.47-3.78), $\mathrm{p}<0.001$ ) were prognostic factors for OS. Similarly, the elderly (HR 1.68, 95\% Cl 1.09-2.59, p=0.018), total gastrectomy (HR 2.16, $95 \% \mathrm{Cl} 1.37-3.38$, $\mathrm{p}<0.001$, stage (HR 4.16, 95\% Cl 2.64-6.54, $\mathrm{p}<0.001$ ), PNI (HR 0.91, 95\% Cl 0.87-0.94, $\mathrm{p}<0.001)$, and PMMA (HR 2.22, 95\% Cl 1.42-3.47, $\mathrm{p}<0.001$ ) were prognostic factors for DFS.

Conclusions: PMMA is suggested as a determinant of OS and DFS in stage I through III GC patients who underwent gastrectomy. Because PMMA is a newly characterized parameter in GC, external validation before clinical applications is a prerequisite.

\section{Background}

In 2017, gastric cancer (GC) was the 5th most common cancer globally, with 1,221,000 incident cases in both sexes. GC also moved from the second leading cause of crude cancer years of life lost to the third place between 2007 and 2017 [1]. However, based on the Korean Central Cancer Registry data (2017), GC was the most common, with 29,685 incident cases in both sexes, and it ranked fourth in terms of the agestandardized mortality rate [2].

Although surgical resection is the most effective treatment for potentially curable GC, radical gastrectomy tends to be associated with high morbidity and mortality. In addition, around $20 \%$ of patients who undergo gastrectomy experience recurrence that may translate into poor survival rates [3]. Therefore, the establishment of biomarkers that can predict postoperative complications, recurrence, and long-term survival are warranted. These may provide clinicians with important preoperative information to improve surgical outcomes. However, biomarkers that have been developed so far are still unsatisfactory [4]. 
The major clinical prognostic factors in GC patients who underwent gastrectomy are as follows: tumornode-metastasis (TNM) stages, tumor size, depth of invasion, surgical margin status [5, 6], lymph node metastasis, peritoneal metastasis, histologic differentiation, and so on [7, 8]. In addition, inflammatory markers, such as absolute monocyte and lymphocyte count prognostic score [9], neutrophil-tolymphocyte ratio (NLR) [10-13], platelet-to-lymphocyte ratio (PLR) [14], and lymphocyte-to-monocyte ratio (LMR) [15], have been suggested as determinants of cancer recurrence and survival.

Recently, the prognostic value of nutritional status has received attention because more than $50 \%$ of GC patients suffer from some degree of malnutrition. In addition, the maintenance of nutrition is an important determinant of postoperative morbidity and mortality [16]. Measuring skeletal muscle mass has been considered as a reliable measure of the nutritional status of cancer patients. Body composition assessment with imaging modalities, including dual-energy X-ray absorptiometry (DEXA), bioelectrical impedance assay (BIA), ultrasound, magnetic resonance imaging (MRI), and computer tomography (CT) scan, can be used to assess skeletal muscle mass and muscle quality in patients with GC.

Along with MRI, CT scan has been considered the gold standard in evaluating muscle mass and quality due to its accuracy, operator independence, reproducibility, and non-invasiveness. As CT scan is a regular part of the standard cancer staging in most cases, additional exposure to radiation doses can be avoided for body composition measurement [17]. However, compared with DEXA, the absence of clear cutoffs to identify low muscle mass, high equipment costs, lack of portability, and complex post-processing limit the clinical application of CT scan [18]. According to the literature, CT scan is more commonly used to assess muscle mass compared to muscle quality, with total abdominal wall musculature as the most common measure for these. Furthermore, the L3 level was the most commonly used landmark in the abdomen [19]. CT-based measurement of the area of skeletal muscle (SMA) is usually performed in the total abdominal wall musculature (i.e., erector spinae, multifidus, quadratus lumborum, psoas, rectus abdominis, external obliques, internal obliques, and transversus abdominis). Skeletal muscle index (SMI), an SMA adjusted for height squared at the L3 level, is considered as a major determinant of muscle mass. According to recent reports, SMI below the threshold has been considered as a risk factor for survival outcomes in patients with gastrointestinal malignancies [20-26]. CT can also detect changes in muscle fat while circumventing the need for an invasive muscle biopsy [27]. This usually involves measuring the intermuscular adipose tissue area or muscle attenuation (MA) [19]. The mean MA within the total abdominal wall musculature (SMMA) at the L3 level has been reported as a determinant of survival in GC patients who underwent gastrectomy [28] and in various gastrointestinal tumors [29-31].

CT-based measurements of the area of paraspinal muscle (PMA) are usually performed, including the multifidus and erector spinae muscles with or without the adjacent muscles (e.g., quadratus lumborum or quadratus lumborum/psoas). The paraspinal muscle index (PMI), a PMA adjusted for height squared at the L3 level, is considered as a major determinant of paraspinal muscle mass. It has been reported as a significant determinant of survival in patients with gastrointestinal malignancies [31-33]. Recently, we reported that the preoperative mean MA within the paraspinal muscle (PMMA) was a significant prognosticator for survival outcome in localized (e.g., stages I and II) GC patients who underwent a 
microscopically margin-negative resection (i.e., R0 resection) [34]. Since there are no validated thresholds for the CT measurements of muscle quantity, more research is required.

Therefore, the purpose of the current study was to evaluate the prognostic potential of the four MRPs (i.e., SMI, SMMA, PMI, and PMMA) using preoperative CT images at the L3 level in a cohort of stage I-III GC patients who underwent $\mathrm{R} 0$ resection.

\section{Methods}

\section{Patients}

Patients who underwent gastric resection for GC between June 2006 and October 2014 at a single university hospital were evaluated retrospectively. The inclusion criteria were as follows: (i) primary GC, according to Lauren's histological classification [35]; (ii) stage I through III, according to the 7th edition of the American Joint Committee (AJCC) on cancer staging [36]; (iii) underwent R0 resection; and (iv) underwent gastrectomy by a gastroenterology expert who participates in more than $50 \mathrm{GC}$ resections annually.

The exclusion criteria were: (i) previous malignancies within the last 5 years, or concurrent second malignancies; (ii) received neoadjuvant chemotherapy, radiation therapy, or other anti-cancer treatment prior to surgery; (iii) stage IV; (iv) absence of RO resection; (v) history of positive tests for human immunodeficiency virus, severe infections within 4 weeks prior to surgery, or active autoimmune diseases that require systemic or immunosuppressive agents; (vi) chronic kidney disease (stage 4 or 5); (vii) chronic obstructive pulmonary disease; (viii) underwent surgical interventions for lumbar spine disorders [37]; (ix) without Korean resident registration number; and ( $\mathrm{x}$ ) without a preoperative abdominal CT scan that can be analyzed.

\section{The baseline clinical characteristics}

Records of demographic (e.g., age, sex, height, body weight, and body mass index [BMI]), and pathological parameters (e.g., tumor location, tumor size, type of gastrectomy, stages, Lauren's histological classification, and lymphatic, vascular, and perineural invasion) were collected and analyzed.

Selected blood tests included peripheral blood leukocyte count, absolute neutrophil count (ANC), absolute monocyte count (AMC), absolute lymphocyte count (ALC), hemoglobin concentration, platelet count, and serum albumin level. Blood test results were analyzed through tests performed within 7 days before surgery. If more than one test result was available, the test result closest to the date of surgery was selected for further analysis. The diagnosis of anemia was based on hemoglobin concentrations below $13 \mathrm{~g} / \mathrm{dL}$ in men and $12 \mathrm{~g} / \mathrm{dL}$ in women. Blood test results were also used to calculate NLR, PLR, LMR, and prognostic nutritional index $(\mathrm{PNI})$. The details of the calculation are as follows: NLR $=($ ANC/ALC) $\times 100$ 
[38]; PLR $=($ platelet counts $/$ ALC $) \times 100$ [38]; LMR $=($ ALC/AMC $) \times 100$ [39]; and PNI $=10 \times$ serum albumin level $(\mathrm{g} / \mathrm{L})+0.005 \times \mathrm{ALC}[40]$.

The muscle-related parameters

Only CT images taken as part of a routine staging work-up within 30 days prior to surgery were analyzed. After identification of the landmark at the L3 level, the corresponding single, enhanced axial CT image was extracted by a musculoskeletal radiologist and was saved as a DICOM image file [41]. The slice thickness in this study was $5 \mathrm{~mm}$. The image was analyzed by a trained nurse using Image ${ }^{\circledR}$ v.1.37, a Java-based image analysis program, which went through an inspection process by a physician who is an expert in the musculoskeletal system.

For measuring the SMA, total abdominal wall musculature was selected as the region of interest (ROI). As for the PMA, a group of muscles, such as the erector spinae, multifidus, quadratus lumborum, and the psoas, were selected as ROIs (Fig. 1). The SMA and PMA were adjusted by the square of height, which produced the SMI and PMI, respectively [42]. In this study, as the muscle mass was evaluated using CT without considering body functions, the term sarcopenia was not applied to mean low SMI or PMI to avoid confusion. To measure the SMMA and PMMA, a Hounsfield unit (HU) threshold range of -29 to + 150 was applied.

\section{Ethical considerations}

This study protocol was approved by the Institutional Review Board of Kyung Hee University Hospital at Gangdong (IRB: 2019-12-039). Written consent was waived because this study was a retrospective study and the lack of it does not negatively affect the rights and well-being of the subject. This study was conducted in accordance with the ethical principles of the Declaration of Helsinki for medical research.

\section{Statistical analysis}

Clinical features were described as medians and interquartile ranges. Either the Mann-Whitney $\mathrm{U}$ test or the Chi-squared test was appropriately used for intergroup comparisons, depending on the nature of the variables. Variables without well-known cutoff points were analyzed as continuous variables without an intentional dichotomy. Disease-free survival (DFS) was defined as the interval from the date of GC surgery to the date of recurrence or death from any cause, whichever came first. Overall survival (OS) was defined as the interval from the date of GC surgery to the date of death from any cause. Patients who did not experience cancer recurrence or death from any cause were censored at the last follow-up. 
The survival rate was estimated using the Kaplan-Meier method, and the statistical significance between survival curves was tested using the log rank test. Statistical significance was declared at $p<0.05$. In addition, the Cox proportional hazards model was used to calculate hazard ratios, which was performed only on variables that met the proportional hazards assumption on the basis of graphic plots of Schoenfeld residuals. Only variables with $p<0.05$ in the univariate analysis were included in the multivariate Cox model. All statistical analyses were performed using $\mathrm{R}$ packages. To measure the model's discriminative capacity, Harrell's concordance index (C-index) for the Cox model was performed. For the diagnosis of multicollinearity of the covariates, the variance inflation factors (VIF) was calculated.

\section{Results}

Demographic and clinical characteristics of patients

The baseline demographic and clinical characteristics of the patients are presented in Table 1. The median age of the patients was 60 years, with more males (65.8\%) than females. There were $207(61.1 \%)$ patients in stage I, 66 (19.5\%) in stage II, and 66 (19.5\%) in stage III. Sixty-eight patients $(20.1 \%)$ underwent total gastrectomy. The intestinal type, according to Lauren's classification, was the most common at $51.9 \%$, and perineural invasion was found in 24 (7.1\%) patients. Anemia was diagnosed in $122(36.0 \%)$ patients, while $21(6.2 \%)$ had hypoalbuminemia with serum albumin levels less than 3.5 $\mathrm{g} / \mathrm{dL}$.

Impact of muscle-related parameters on the Kaplan-Meier curves

Because there were significant differences in the medians of the four MRPs (i.e., SMI, SMMA, PMI, and PMMA) between sexes ( $p<0.001$ in all variables), they were dichotomized for each sex, using thresholds determined using the receiver operating characteristic curve analysis (Table 2). The number of patients below the SMI and SMMA thresholds were 143 (42.2\%) and 111 (32.7\%), respectively. In addition, the number of patients below the PMI and PMMA thresholds were 178 (52.5\%) and 137 (40.4\%).

When evaluating the four MRPs using the Kaplan-Meier method, there were significant differences in OS in variables, such as the SMMA $(p<0.001), \mathrm{PMI}(p=0.027)$, and PMMA $(p<0.001)$. The five-year OS rates were $70.2 \%$ in the low SMMA group and $85.5 \%$ in the high SMMA group. In addition, the five-year OS rates were $71.5 \%$ in the low PMMA group and $86.6 \%$ in the high PMMA group. As for DFS, there were significant differences in DFS in variables, such as the SMMA $(p<0.001)$ and PMMA $(p<0.001)$. The fiveyear DFS rates were $64.8 \%$ in the low SMMA group and $83.7 \%$ in the high SMMA group. In addition, the five-year DFS rates were $66.3 \%$ in the low PMMA group and $85.1 \%$ in the high PMMA group (Fig. 2).

Cox proportional hazard model of the risk factors on OS and DFS 
Significant variables in the univariate Cox proportional hazard model for OS were the elderly, total gastrectomy, tumor size, tumor stage, lymphatic invasion, vascular invasion, perineural invasion, anemia, NLR, PLR, LMR, PNI, SMMA, PMI, and PMMA. Multivariate survival analysis using Cox's model revealed the following significant variables: the elderly (hazard ratio [HR] 2.08, 95\% confidence interval [CI] 1.30$3.32, p=0.002)$, total gastrectomy (HR 2.14, 95\% Cl 1.32-3.48, $p=0.002)$, stage (HR 3.41, 95\% Cl 2.10$5.54, p<0.001$ ), perineural invasion (HR 2.01, 95\% Cl 1.02-3.93, $p=0.042)$, PNI (HR 0.91, 95\% $\mathrm{Cl} 0.88-0.95$, $p<0.001$ ), and PMMA (HR 2.36, 95\% Cl 1.47-3.78), $p<0.001$ ). Harrell's C-index for the Cox model to measure the model's discriminative capacity was 0.822 , indicating excellent discrimination. In addition, the VIF was calculated for diagnosis of multicollinearity of the covariates. The VIFs for the elderly, total gastrectomy, stage, perineural invasion, PNI, and PMMA were 1.10, 1.11, 1.17, 1.17, 1.10, and 1.06, respectively. Therefore, there was no significant association between the covariates (Table 3 ).

Except for PMI, the same variables that were found to be significant in the OS analysis using the univariate Cox model were also found to be significant in the DFS analysis. Multivariate survival analysis using Cox's model revealed that the elderly (HR 1.68, 95\% Cl 1.09-2.59, $p=0.018$ ), total gastrectomy (HR $2.16,95 \% \mathrm{Cl}, 1.37-3.38, p<0.001)$, stage (HR 4.16, 95\% Cl 2.64-6.54, $p<0.001)$, PNI (HR 0.91, $95 \% \mathrm{Cl}$, $0.87-0.94, p<0.001$ ), and PMMA (HR 2.22, 95\% Cl 1.42-3.47, $p<0.001$ ) were significant prognostic factors for DFS. Harrell's C-index was 0.817 , suggesting excellent discrimination. In addition, the VIFs for the elderly, total gastrectomy, stage, PNI, and PMMA were 1.03, 1.04, 1.11, 1.11, and 1.04, respectively. Therefore, there was no significant association between the covariates (Table 4).

\section{Discussion}

The purpose of the current study was to evaluate the clinical significance of the four MRPs (i.e., SMI, SMMA, PMI, and PMMA) at the L3 level in stage I through III GC patients who underwent R0 resection. In this study, using a multivariate Cox model, the PMMA was the only MRP that proved to be a significant determinant of OS and DFS.

In this study, the SMA at the L3 level was correlated with height, as in Yoshizumi's previous study. [43]. Therefore, we calculated the SMI, the SMA normalized for height squared, for use as an indicator of muscle mass. Although the most frequent cutoffs of SMI were $52-55 \mathrm{~cm}^{2} / \mathrm{m}^{2}$ for men and $39-41$ $\mathrm{cm}^{2} / \mathrm{m}^{2}$ for women in the literature [19], we determined the cutoffs based on our patient cohort. As the SMI was sex-dependent, it was dichotomized as low and high groups with sex-specific cutoffs (i.e., 46.48 $\mathrm{cm}^{2} / \mathrm{m}^{2}$ for men and $40.77 \mathrm{~cm}^{2} / \mathrm{m}^{2}$ for women). However, it was not an important determinant of OS or DFS when using the Cox model. In addition, even when SMI was dichotomized as low and high groups with sex-specific cutoffs according to Martin et al. (i.e., $53 \mathrm{~cm}^{2} / \mathrm{m}^{2}$ for men with BMI of $25 \mathrm{~cm}^{2} / \mathrm{m}^{2}$ or higher, $43 \mathrm{~cm}^{2} / \mathrm{m}^{2}$ for men with a BMI lower than $25 \mathrm{~cm}^{2} / \mathrm{m}^{2}$, and $41 \mathrm{~cm}^{2} / \mathrm{m}^{2}$ for women, irrespective of $\mathrm{BMI}$ ), it was still not an important determinant of OS or DFS [29]. Thus, the results of the current study were somewhat inconsistent with previous studies that found that the SMI is an important determinant of OS in GC patients who underwent gastrectomy [22-26]. However, in a recent study by Hacker et al., 
encompassing 761 advanced stage GC patients, the SMI was also not a prognostic factor for OS [31]. In addition, in Zhuang et al.'s study, encompassing 937 stage I GC patients, it was not associated with OS [25]. The reason for the discrepancies between studies is unclear, but the differences in cutoffs, pathologic stage, ethnicity, and variation in CT acquisition parameters (e.g., type of CT examination, slice thickness, and administration of contrast media) and image analysis software may have possibly caused these [19]. Because only a limited number of studies have been performed on CT-based SMI measurements in GC patients, further studies are needed to validate the prognostic role of the SMI.

In addition to the SMA, we measured the PMA at the L3 level as another marker of muscle mass. In our study, the group of muscles, including the erector spinae, multifidus, psoas, and quadratus lumborum, was defined as the paraspinal muscle, as had been reported previously [29, 34, 44, 45]. Since the PMA correlated with height, we calculated the PMI, the PMA normalized for height squared for statistical analysis. As PMI was sex-dependent, PMI was dichotomized as low and high groups with sex-specific cutoffs ( $26.78 \mathrm{~cm}^{2} / \mathrm{m}^{2}$ for men and $23.80 \mathrm{~cm}^{2} / \mathrm{m}^{2}$ for women). Using the multivariate Cox model, PMI was not an important determinant of OS or DFS in our study. Similarly, in Hacker's recent study on advanced gastric and gastroesophageal junction cancer, while the PMI was an important determinant of OS ( $p=0.003)$, DFS was not significant using a multivariate Cox model [31]. Therefore, our findings are in line with previous studies in terms of DFS, but not OS. The reason for the inconsistent findings between studies is not certain; however, the differences in definition of paraspinal muscles (i.e., with or without the psoas muscle), cutoffs, stage of the tumor (i.e., stage I to III vs. stage IV), tumor location, and ethnicity (i.e., Asian vs. international) may have affected the differences in outcomes between studies.

Fat in the skeletal muscles is present in the form of intermuscular adipose tissue, intramuscular adipose tissue, or intramyocellular lipids [46]. The MA, which is calculated using CT images, is a radiologic index of muscle fat content; the values of the mean MA are inversely correlated with muscle fat content [27]. Under physiological conditions, the values of the MA are considered dependent on sex, age, and ethnicity [47]. Excess fat deposition in skeletal muscle, a pathological variation in the MA, is observed in patients with malignant tumors [27].

In previous studies on gastrointestinal malignancies, the SMMA was usually measured within the total abdominal wall musculature at the L3 level with predefined $\mathrm{HU}$ ranges of $-29 \mathrm{HU}$ to $+150 \mathrm{HU}$. Therefore, in our study, the SMMA was dichotomized using sex-specific cutoffs (i.e., $40.56 \mathrm{HU}$ for men and $26.39 \mathrm{HU}$ for women), and the adjustment for age was performed by including age as a covariate at entry [48]. In this study, while the SMMA was found to be an important determinant of OS and DFS in the univariate Cox model, but not in the multivariate model. Similarly, in Tamandl et al.'s study, the SMMA was a determinant of OS in patients with resected esophageal or gastroesophageal junction cancer by applying the univariate Cox model, but was no longer true in the multivariate Cox model; therefore, their findings are consistent with our findings [30]. On the other hand, in other studies, using the multivariate Cox model, the SMMA was a significant prognostic factor for determining OS in patients with GC who underwent gastrectomy, patients with various gastrointestinal malignancies, and patients with gastric or 
gastroesophageal junction cancer. Therefore, the results of these studies were inconsistent with our findings $[28,29,31]$.

In our study, the PMMA was measured in a group of skeletal muscles (i.e., erector spinae, multifidus, quadratus lumborum, and psoas muscle) at the L3 level with predefined HU ranges of $-29 \mathrm{HU}$ to $+150 \mathrm{HU}$. Before statistical evaluation, this was dichotomized with sex-specific cutoffs ( $48.12 \mathrm{HU}$ for men and 34.24 HU for women), and the adjustment for age was performed by including age as a covariate at entry. Using the multivariate Cox model, the PMMA was a significant determinant of both OS and DFS. The results of this study were consistent with our group's previous study that enrolled only stage I and II GC without including SMMA as a covariate [34]. Similarly, in Dohzono's study of patients with gastrointestinal cancers, including GC at 25\%, the PMMA was a significant determinant of survival. However, discrepancies in terms of the cutoffs, paraspinal muscle definition, tumor stage, and tumor histology limit the exact comparison between studies [33].

As a subgroup analysis, the multivariate Cox model with or without the PMMA was applied. When applying multivariate Cox analysis while excluding the PMMA as a covariate in this study, the SMMA was an independent determinant of OS $(p=0.008)$ and DFS $(p=0.008)$. In addition, Harrell's C-index for the multivariate Cox model excluding PMMA was 0.816 and 0.810 for OS and DFS, respectively. In comparison, when the PMMA was included as a covariate, Harrell's C-index increased to 0.822 for OS and 0.817 for DFS. Therefore, the SMMA is considered a significant determinant of OS and DFS, and its statistical significance was attenuated by adding the PMMA as a covariate to the multivariate Cox model. Therefore, these findings show that the PMMA rather than the SMMA may be a more accurate determinant of survival in GC patients undergoing gastrectomy. It is also a time-saving strategy because the ROIs for the PMMA are apparently smaller than those of the SMMA. In addition, while the PMMA was an important determinant of OS and DFS, upon application of the multivariate Cox model, the PMI was not a determinant of survival. Thus, muscle quality (i.e., PMMA) appears to be a more powerful factor in determining survival in our patient cohort compared to muscle mass (i.e., PMI). Similarly, in Dohzono et al.'s study, while the PMMA was an important determinant of OS, the PMI was not an important determinant of OS, using the multivariate Cox model [33]. In addition, Hacker et al. found that, although the SMMA was an important determinant of OS, the SMI was not an important determinant of OS, upon application of the multivariate Cox model [31]. On the other hand, in studies by Zhuang [28] and Martin [29], both the SMI and SMMA were significant determinants of OS. Therefore, the results of these studies were inconsistent with our finding, which suggests that muscle quality may be a stronger determinant than muscle mass. Since studies on MA, including PMMA along with SMMA, are rare, more studies are needed to verify the clinical significance of MA as a survival determinant.

In our study, multivariate Cox analysis revealed that total gastrectomy was an important determinant of OS and DFS. Compared with subtotal gastrectomy, total gastrectomy is a technically difficult surgery associated with poor prognosis due to high postoperative complications, infection rates, and hospitalization rates $[49,50]$. Therefore, our findings confirmed the current trend. In this study, we also found that perineural invasion was an independent determinant of survival by applying a multivariate 
Cox model. The prognostic value of perineural invasion has been reported previously [51]. In the present study, the PNI, which is calculated based on serum albumin levels and peripheral ALC, was an important determinant of OS and DFS in the multivariate Cox model [40]. The clinical role of PNI as an indicator of nutritional and immunological status in cancer patients has been validated. Several previous studies on GC have confirmed that the PNI is associated with the depth of invasion, lymph node metastasis, pathological stage, and lymph and vascular infiltration and that low PNI was an adverse prognosticator for survival [51-53].

The strengths of our study are as follows: First, this was the first study to assess the prognostic significance of the PMMA, along with the SMMA as a covariate, in patients with stage I to III GC undergoing curative gastrectomy. In this study, we found that the PMMA was the only MRP capable of predicting OS and DFS by applying a multivariate Cox model. Thus, skeletal muscle quality (i.e., PMMA) compared to skeletal muscle mass (i.e., SMI or PMI) appears to be a more important determinant of survival. Second, for MRP measurements, we used CT images taken as a part of standard cancer staging to avoid additional costs and radiation exposure. Finally, for consistency, we included only those patients who underwent curative gastrectomy by a gastroenterology expert.

However, since this study had several limitations, the results of the study should be interpreted carefully. First, this study was performed retrospectively; therefore, omission of data including CT images is inevitable and may have affected the results. Second, although random errors and potential biases were controlled from the study design to implementation, the lack of external validation was another limitation of our study. For verification, public databases were investigated in domestic and global databases, but there were no CT images suitable for measuring the MRPs. However, based on the results of this study, it is possible to conduct a prospective study with an independent external validation group at the next stage of this study, which could be an essential step for the clinical use of PMMA. Third, since it was a retrospective study, there was no opportunity to provide special interventions to improve postoperative outcomes in patients with PMMA below the threshold. Finally, the cutoffs for dichotomization of MRPs in our cohort were determined using receiver operating characteristic curve analysis. As such, the cutoffs presented in this study may not be applicable to patients with malignant tumors other than GC.

In conclusion, we found that the PMMA was a significant determinant of OS and DFS. Since the PMMA is a newly characterized determinant of survival in GC, its prognostic importance requires further validation prior to clinical application. In addition, measurements of muscle mass and MA using CT require standardization for comparison between studies.

\section{Tables}

Table 1 Characteristic of patients. IQR: interquartile range; BMI: body mass index; TNM: tumor-node-metastasis; WBC: white blood cell; NLR: neutrophil-to-lymphocyte ratio; PLR: platelet-to-lymphocyte ratio; LMR: lymphocyte-to-monocyte ratio; PNI: prognostic nutritional index 


\begin{tabular}{|c|c|}
\hline \multirow{2}{*}{\multicolumn{2}{|c|}{$\frac{\text { Median (IQR): or } n(\%)}{60.0(52.0-69.0)}$}} \\
\hline & \\
\hline Gender Male & $223(65.8 \%)$ \\
\hline Female & $\begin{array}{l}223(05.0 \%) \\
116(34.2 \%)\end{array}$ \\
\hline $\operatorname{BMI}\left(\mathrm{kg} / \mathrm{m}^{2}\right)$ & $23.7(21.4-25.8)$ \\
\hline \multicolumn{2}{|l|}{ Site of tumor } \\
\hline Upper & $32(9.4 \%)$ \\
\hline Middle & $139(41.0 \%)$ \\
\hline Lower & $164(48.4 \%)$ \\
\hline Diffuse & $4(1.2 \%)$ \\
\hline Size of tumor $(\mathrm{cm})$ & $3.0(2.0-5.2)$ \\
\hline \multicolumn{2}{|l|}{ Nodal invasion } \\
\hline No & $223(65.8 \%)$ \\
\hline Yes & $116(34.2 \%)$ \\
\hline \multicolumn{2}{|l|}{ TNM stage } \\
\hline I & $207(61.1 \%)$ \\
\hline II & $66(19.5 \%)$ \\
\hline III & $66(19.5 \%)$ \\
\hline \multicolumn{2}{|l|}{ Gastrectomy } \\
\hline Partial & $271(79.9 \%)$ \\
\hline Total & $68(20.1 \%)$ \\
\hline \multicolumn{2}{|l|}{ Lauren classification } \\
\hline Intestinal & 176 (51.9\%) \\
\hline Diffuse & $81(23.9 \%)$ \\
\hline Mixed & $68(20.1 \%)$ \\
\hline Unknown & $14(4.1 \%)$ \\
\hline \multicolumn{2}{|l|}{ Lymphatic invasion } \\
\hline No & $231(68.1 \%)$ \\
\hline Yes & $108(31.9 \%)$ \\
\hline \multicolumn{2}{|l|}{ Vascular invasion } \\
\hline No & $322(95.0 \%)$ \\
\hline Yes & $17(5.0 \%)$ \\
\hline \multicolumn{2}{|l|}{ Perineural invasion } \\
\hline No & $315(92.9 \%)$ \\
\hline Yes & $24(7.1 \%)$ \\
\hline WBC (per $\mu \mathrm{L})$ & $6500(5400-8000)$ \\
\hline \multicolumn{2}{|l|}{ Anemia* } \\
\hline No & $217(64.0 \%)$ \\
\hline Yes & $122(36.0 \%)$ \\
\hline Platelet $\left(10^{3} / \mu \mathrm{L}\right)$ & $236(203-278)$ \\
\hline Serum albumin (g/dL) & $4.1(3.9-4.3)$ \\
\hline NLR & $2.0(1.4-2.6)$ \\
\hline PLR & $120.9(96.0-154.1)$ \\
\hline LMR & $4.4(3.3-5.5)$ \\
\hline PNI & $51.0(47.8-54.8)$ \\
\hline \multicolumn{2}{|l|}{ Adjuvant chemotherapy } \\
\hline No & $215(63.4 \%)$ \\
\hline Yes & $124(36.6 \%)$ \\
\hline
\end{tabular}

* The cutoff points are $12 \mathrm{~g} / \mathrm{dL}$ in female patients and $13 \mathrm{~g} / \mathrm{dL}$ in male patients. 
Table 2 The medians and threshold values of muscle-related parameters according to gender. IQR: interquartile range; SMA: skeletal muscle area; SMI: skeletal muscle index; SMMA: mean attenuation within total abdominal wall musculature; PMA: paraspinal muscle area; PMI: paraspinal muscle index; PMMA: mean attenuation within paraspinal muscle

\begin{tabular}{|c|c|c|c|c|c|}
\hline & \multicolumn{3}{|c|}{ Median (IQR) } & \multicolumn{2}{|c|}{ Threshold values* } \\
\hline & Male & Female & & Male & Female \\
\hline & $(n=223)$ & $(n=116)$ & $p$-value & $(n=223)$ & $(n=116)$ \\
\hline SMA $\left(\mathrm{cm}^{2}\right)$ & $139.8(122.9-152.6)$ & $93.2(84.7-100.8)$ & $<0.001$ & 122.8 & 80.45 \\
\hline $\operatorname{SMI}\left(\mathrm{cm}^{2} / \mathrm{m}^{2}\right)$ & $50.2(44.8-55.1)$ & $39.2(36.3-42.4)$ & $<0.001$ & 46.48 & 40.77 \\
\hline SMMA (HU) & $42.5(35.4-47.6)$ & $37.3(30.1-42.7)$ & $<0.001$ & 40.56 & 26.39 \\
\hline PMA $\left(\mathrm{cm}^{2}\right)$ & $76.1(67.6-85.1)$ & $52.7(47.7-57.5)$ & $<0.001$ & 62.42 & 47.97 \\
\hline PMI $\left(\mathrm{cm}^{2} / \mathrm{m}^{2}\right)$ & $27.6(24.8-30.2)$ & $22.2(19.9-24.0)$ & $<0.001$ & 26.78 & 23.80 \\
\hline PMMA (HU) & 48.1 (41.2-53.3) & $43.7(35.4-49.3)$ & $<0.001$ & 48.12 & 34.24 \\
\hline
\end{tabular}

* The threshold is determined using the receiver operating characteristic curve.

Table 3 Univariate and multivariate Cox proportional hazards regression analysis of overall survival. HR: hazard ratio; Cl: confidence interval; BMI: body mass index; TNM: tumor-node-metastasis; NLR: neutrophil-to-lymphocyte ratio; PLR: platelet-to-lymphocyte ratio; LMR: lymphocyte-to-monocyte ratio; PNI: prognostic nutritional index; SMI: skeletal muscle index; SMMA: mean attenuation within total abdominal wall musculature; PMI: paraspinal muscle index; PMMA: mean attenuation within paraspinal muscle. 


\begin{tabular}{|c|c|c|c|c|c|}
\hline \multirow[t]{3}{*}{ Variable } & \multicolumn{5}{|c|}{ Overall survival } \\
\hline & Univariat & & Multivaria & & \\
\hline & HR (95\% CI) & $p$-value & HR (95\% CI) & $p$-value & \\
\hline Elderly (yes vs. no)* & $2.15(1.59-$ & $<0.001$ & $2.08(1.30-$ & 0.002 & \\
\hline Gender (female vs. male) & $0.78(0.49-$ & 0.316 & & & \\
\hline $\operatorname{BMI}\left(\mathrm{kg} / \mathrm{m}^{2}\right)$ & $0.97(0.90-$ & 0.329 & & & - \\
\hline Total gastrectomy (yes vs. no) & $2.95(1.88-$ & $<0.001$ & $2.14(1.32-$ & 0.002 & - \\
\hline Size of tumor $(\mathrm{cm})$ & $1.17(1.12-$ & $<0.001$ & $\begin{array}{r}0.40) \\
-\end{array}$ & - & \\
\hline TNM stage (III vs. I/II) & $5.38(3.46-$ & $<0.001$ & $3.41(2.10-$ & $<0.001$ & \\
\hline Histology (intestinal vs. & $0.91(0.59-$ & 0.678 & 0 & - & \\
\hline Lymphatic invasion (yes vs. no) & $3.29(2.11-$ & $<0.001$ & - & - & \\
\hline Vascular invasion (yes vs. no) & $3.34(1.67-$ & 0.007 & & & \\
\hline Perineural invasion (yes vs. no) & $2.76(1.49-$ & 0.002 & $2.01(1.02-$ & 0.042 & \\
\hline Anemia (yes vs. no) ${ }^{* * *}$ & $3.10(1.98-$ & $<0.001$ & $\begin{array}{r}0.90) \\
-\end{array}$ & - & - \\
\hline NLR & $1.28(1.15-$ & $<0.001$ & - & - & - \\
\hline PLR & $1.01(1.00-$ & $<0.001$ & - & - & \\
\hline LMR & $0.74(0.62-$ & 0.003 & & & - \\
\hline PNI & $0.87(0.84-$ & $<0.001$ & $0.91(0.88-$ & $<0.001$ & \\
\hline SMI (low vs. high) & $1.42(0.92-$ & 0.117 & - & - & \\
\hline SMMA (low vs. high) & $2.75(1.77-$ & $<0.001$ & - & - & - \\
\hline PMI (low vs. high) & $1.66(1.05-$ & 0.029 & - & - & - \\
\hline PMMA (low vs. high) & $2.81\left(\begin{array}{c}(1.78- \\
4.44)\end{array}\right.$ & $<0.001$ & 2.36 (1.47- & $<0.001$ & - \\
\hline
\end{tabular}

* The cutoff point is 65 years.

** Lauren classification

*** The cutoff points are $12 \mathrm{~g} / \mathrm{dL}$ in female patients and $13 \mathrm{~g} / \mathrm{dL}$ in male patients.

Table 4 Univariate and multivariate Cox proportional hazards regression analysis of disease-free survival. HR: hazard ratio; CI: confidence interval; BMI: body mass index; 
TNM: tumor-node-metastasis; NLR: neutrophil-to-lymphocyte ratio; PLR: platelet-tolymphocyte ratio; LMR: lymphocyte-to-monocyte ratio; PNI: prognostic nutritional index; SMI: skeletal muscle index; SMMA: mean attenuation within total abdominal wall musculature; PMI: paraspinal muscle index; PMMA: mean attenuation within paraspinal muscle.

\begin{tabular}{|c|c|c|c|c|}
\hline \multirow[t]{3}{*}{ Variable } & \multicolumn{4}{|c|}{ Disease-free survival } \\
\hline & \multicolumn{2}{|c|}{ Univariate } & \multicolumn{2}{|c|}{ Multivariate } \\
\hline & $\mathrm{HR}(95 \% \mathrm{CI})$ & $p$-value & HR (95\% CI) & $p$-value \\
\hline Elderly (yes vs. no)* & $2.33(1.53-$ & $<0.001$ & $1.68(1.09-$ & 0.018 \\
\hline Gender (female vs. male) & $0.67(0.42-$ & 0.100 & & \\
\hline BMI $\left(\mathrm{kg} / \mathrm{m}^{2}\right)$ & $0.97(0.91-$ & 0.339 & & \\
\hline Total gastrectomy (yes vs. no) & $2.72(1.76-$ & $<0.001$ & $2.16(1.37-$ & $<0.001$ \\
\hline Size of tumor $(\mathrm{cm})$ & $1.17(1.12-$ & $<0.001$ & & \\
\hline TNM stage (III vs. I/II) & $5.88(3.86-$ & $<0.001$ & $4.16(2.64-$ & $<0.001$ \\
\hline Histology (intestinal vs. & $0.91(0.60-$ & 0.651 & & \\
\hline Lymphatic invasion (yes vs. no) & $3.16(2.07-$ & $<0.001$ & & \\
\hline Vascular invasion (yes vs. no) & $3.74(1.93-$ & $<0.001$ & & \\
\hline Perineural invasion (yes vs. no) & $2.51(1.36-$ & 0.032 & & \\
\hline Anemia (yes vs. no) $* * *$ & $2.87(1.88-$ & $<0.001$ & & \\
\hline NLR & $1.31(1.18-$ & $<0.001$ & & \\
\hline PLR & $1.01(1.01-$ & $<0.001$ & & \\
\hline LMR & $0.71(0.60-$ & $<0.001$ & & \\
\hline PNI & $0.87(0.84-$ & $<0.001$ & $0.91(0.87-$ & $<0.001$ \\
\hline SMI (low vs. high) & $1.27(0.83-$ & 0.271 & & \\
\hline SMMA (low vs. high) & $2.90(1.90-$ & $<0.001$ & & \\
\hline PMI (low vs. high) & $1.39(0.91-$ & 0.130 & & \\
\hline PMMA (low vs. high) & $\begin{array}{l}2.85(1.85- \\
4.41)\end{array}$ & $<0.001$ & $\begin{array}{l}2.22(1.42- \\
3.47)\end{array}$ & $<0.001$ \\
\hline
\end{tabular}

* The cutoff point is 65 years.

** Lauren classification 
*** The cutoff points are $12 \mathrm{~g} / \mathrm{dL}$ in female patients and $13 \mathrm{~g} / \mathrm{dL}$ in male patients.

\section{Declarations}

Acknowledgements

Funding

This research received no specific grant from any funding agency in the public: commercial: or not-forprofit sectors.

Competing Interests

The authors have declared that no competing interest exists.

\section{References}

1. Fitzmaurice C: Abate D: Abbasi N: Abbastabar H: Abd-Allah F: Abdel-Rahman O: Abdelalim A: Abdoli A: Abdollahpour l: Abdulle ASM et al: Global: Regional: and National Cancer Incidence: Mortality: Years of Life Lost: Years Lived With Disability: and Disability-Adjusted Life-Years for 29 Cancer Groups: 1990 to 2017: A Systematic Analysis for the Global Burden of Disease Study. JAMA oncology 2019: 5(12):1749-1768.

2. Hong S: Won YJ: Park YR: Jung KW: Kong HJ: Lee ES: Cancer Statistics in Korea: Incidence: Mortality: Survival: and Prevalence in 2017. Cancer research and treatment : official journal of Korean Cancer Association 2020: 52(2):335-350.

3. Dittmar Y: Schüle S: Koch A: Rauchfuss F: Scheuerlein H: Settmacher U: Predictive factors for survival and recurrence rate in patients with node-negative gastric cancer-a European single-centre experience. Langenbeck's archives of surgery 2015: 400(1):27-35.

4. Correia MI: Waitzberg DL: The impact of malnutrition on morbidity: mortality: length of hospital stay and costs evaluated through a multivariate model analysis. Clinical nutrition (Edinburgh: Scotland) 2003: 22(3):235-239.

5. Siewert JR: Bottcher K: Stein HJ: Roder JD: Relevant prognostic factors in gastric cancer: ten-year results of the German Gastric Cancer Study. Annals of surgery 1998: 228(4):449-461.

6. Dittmar Y: Schule S: Koch A: Rauchfuss F: Scheuerlein H: Settmacher U: Predictive factors for survival and recurrence rate in patients with node-negative gastric cancer-a European single-centre experience. Langenbeck's archives of surgery 2014. 
7. Arrington AK: Nelson R: Patel SS: Luu C: Ko M: Garcia-Aguilar J: Kim J: Timing of chemotherapy and survival in patients with resectable gastric adenocarcinoma. World journal of gastrointestinal surgery 2013: 5(12):321-328.

8. Dutta S: Crumley AB: Fullarton GM: Horgan PG: McMillan DC: Comparison of the prognostic value of tumour and patient related factors in patients undergoing potentially curative resection of gastric cancer. American journal of surgery 2012: 204(3):294-299.

9. Eo WK: Jeong DW: Chang HJ: Won KY: Choi SI: Kim SH: Chun SW: Oh YL: Lee TH: Kim YO et al: Absolute monocyte and lymphocyte count prognostic score for patients with gastric cancer. World journal of gastroenterology 2015: 21(9):2668-2676.

10. Shimada H: Takiguchi N: Kainuma O: Soda H: Ikeda A: Cho A: Miyazaki A: Gunji H: Yamamoto H: Nagata M: High preoperative neutrophil-lymphocyte ratio predicts poor survival in patients with gastric cancer. Gastric cancer : official journal of the International Gastric Cancer Association and the Japanese Gastric Cancer Association 2010: 13(3):170-176.

11. Lee DY: Hong SW: Chang YG: Lee WY: Lee B: Clinical significance of preoperative inflammatory parameters in gastric cancer patients. Journal of gastric cancer 2013: 13(2):111-116.

12. Lee S: Oh SY: Kim SH: Lee JH: Kim MC: Kim KH: Kim HJ: Prognostic significance of neutrophil lymphocyte ratio and platelet lymphocyte ratio in advanced gastric cancer patients treated with FOLFOX chemotherapy. BMC Cancer 2013: 13:350.

13. Mohri Y: Tanaka K: Ohi M: Yokoe T: Miki C: Kusunoki M: Prognostic significance of host- and tumorrelated factors in patients with gastric cancer. World journal of surgery 2010: 34(2):285-290.

14. Ohe Y: Fushida S: Yamaguchi T: Kinoshita J: Saito H: Okamoto K: Nakamura K: Tajima H: Ninomiya I: Ohta T: Peripheral Blood Platelet-Lymphocyte Ratio Is Good Predictor of Chemosensitivity and Prognosis in Gastric Cancer Patients. Cancer management and research 2020: 12:1303-1311.

15. Ma JY: Liu Q: Clinicopathological and prognostic significance of lymphocyte to monocyte ratio in patients with gastric cancer: A meta-analysis. International journal of surgery (London: England) 2018: 50:67-71.

16. Karcz W: Gluszek S: Kot M: Matykiewicz J: Influence of nutritional treatment on the postoperative course in patients with gastric cancer. Adv Med Sci 2006: 51:278-282.

17. Kuwada K: Kuroda S: Kikuchi S: Yoshida R: Nishizaki M: Kagawa S: Fujiwara T: Sarcopenia and Comorbidity in Gastric Cancer Surgery as a Useful Combined Factor to Predict Eventual Death from Other Causes. Annals of surgical oncology 2018: 25(5):1160-1166.

18. Albano D: Messina C: Vitale J: Sconfienza LM: Imaging of sarcopenia: old evidence and new insights. European radiology 2020: 30(4):2199-2208.

19. Amini B: Boyle SP: Boutin RD: Lenchik L: Approaches to Assessment of Muscle Mass and Myosteatosis on Computed Tomography: A Systematic Review. The journals of gerontology Series A: Biological sciences and medical sciences 2019: 74(10):1671-1678.

20. Su H: Ruan J: Chen T: Lin E: Shi L: CT-assessed sarcopenia is a predictive factor for both long-term and short-term outcomes in gastrointestinal oncology patients: a systematic review and meta- 
analysis. Cancer Imaging 2019: 19(1):82.

21. Kamarajah SK: Bundred J: Tan BHL: Body composition assessment and sarcopenia in patients with gastric cancer: a systematic review and meta-analysis. Gastric cancer : official journal of the International Gastric Cancer Association and the Japanese Gastric Cancer Association 2019: 22(1):10-22.

22. Sakurai K: Kubo N: Tamura T: Toyokawa T: Amano R: Tanaka H: Muguruma K: Yashiro M: Maeda K: Hirakawa $\mathrm{K}$ et al: Adverse Effects of Low Preoperative Skeletal Muscle Mass in Patients Undergoing Gastrectomy for Gastric Cancer. Annals of surgical oncology 2017: 24(9):2712-2719.

23. Kim EY: Jun KH: Kim SY: Chin HM: Body mass index and skeletal muscle index are useful prognostic factors for overall survival after gastrectomy for gastric cancer: Retrospective cohort study. Medicine 2020: 99(47):e23363.

24. Zheng ZF: Lu J: Xie JW: Wang JB: Lin JX: Chen QY: Cao LL: Lin M: Tu RH: Zheng CH et al: Preoperative skeletal muscle index vs the controlling nutritional status score: Which is a better objective predictor of long-term survival for gastric cancer patients after radical gastrectomy? Cancer medicine 2018: 7(8):3537-3547.

25. Zhuang CL: Huang DD: Pang WY: Zhou CJ: Wang SL: Lou N: Ma LL: Yu Z: Shen X: Sarcopenia is an Independent Predictor of Severe Postoperative Complications and Long-Term Survival After Radical Gastrectomy for Gastric Cancer: Analysis from a Large-Scale Cohort. Medicine 2016: 95(13):e3164.

26. Borggreve AS: den Boer RB: van Boxel Gl: de Jong PA: Veldhuis WB: Steenhagen E: van Hillegersberg R: Ruurda JP: The Predictive Value of Low Muscle Mass as Measured on CT Scans for Postoperative Complications and Mortality in Gastric Cancer Patients: A Systematic Review and Meta-Analysis. Journal of clinical medicine 2020: 9(1).

27. Aubrey J: Esfandiari N: Baracos VE: Buteau FA: Frenette J: Putman CT: Mazurak VC: Measurement of skeletal muscle radiation attenuation and basis of its biological variation. Acta physiologica (Oxford: England) 2014: 210(3):489-497.

28. Zhuang CL: Shen X: Huang YY: Zhang FM: Chen XY: Ma LL: Chen XL: Yu Z: Wang SL: Myosteatosis predicts prognosis after radical gastrectomy for gastric cancer: A propensity score-matched analysis from a large-scale cohort. Surgery 2019: 166(3):297-304.

29. Martin L: Birdsell L: Macdonald N: Reiman T: Clandinin MT: McCargar LJ: Murphy R: Ghosh S: Sawyer MB: Baracos VE: Cancer cachexia in the age of obesity: skeletal muscle depletion is a powerful prognostic factor: independent of body mass index. J Clin Oncol 2013: 31(12):1539-1547.

30. Tamandl D: Paireder M: Asari R: Baltzer PA: Schoppmann SF: Ba-Ssalamah A: Markers of sarcopenia quantified by computed tomography predict adverse long-term outcome in patients with resected oesophageal or gastro-oesophageal junction cancer. European radiology 2016: 26(5):1359-1367.

31. Hacker UT: Hasenclever D: Linder N: Stocker G: Chung HC: Kang YK: Moehler M: Busse H: Lordick F: Prognostic role of body composition parameters in gastric/gastroesophageal junction cancer patients from the EXPAND trial. J Cachexia Sarcopenia Muscle 2020: 11(1):135-144. 
32. Deng CY: Lin YC: Wu JS: Cheung YC: Fan CW: Yeh KY: McMahon CJ: Progressive Sarcopenia in Patients With Colorectal Cancer Predicts Survival. AJR Am J Roentgeno/2018: 210(3):526-532.

33. Dohzono S: Sasaoka R: Takamatsu K: Hoshino M: Nakamura H: Prognostic value of paravertebral muscle density in patients with spinal metastases from gastrointestinal cancer. Support Care Cancer 2019: 27(4):1207-1213.

34. Eo W: Kwon J: An S: Lee S: Kim S: Nam D: Han GY: Choi SI: Chung HY: Clinical Significance of Paraspinal Muscle Parameters as a prognostic factor for survival in Gastric Cancer Patients who underwent Curative Surgical Resection. Journal of Cancer 2020: 11(19):5792-5801.

35. Lauren P: THE TWO HISTOLOGICAL MAIN TYPES OF GASTRIC CARCINOMA: DIFFUSE AND SOCALLED INTESTINAL-TYPE CARCINOMA. AN ATTEMPT AT A HISTO-CLINICAL CLASSIFICATION. Acta pathologica et microbiologica Scandinavica 1965: 64:31-49.

36. Santiago JM: Sasako M: Osorio J: [TNM-7th edition 2009 (UICC/AJCC) and Japanese Classification 2010 in Gastric Cancer. Towards simplicity and standardisation in the management of gastric cancer]. Cirugia espanola 2011: 89(5):275-281.

37. Hicks GE: Simonsick EM: Harris TB: Newman AB: Weiner DK: Nevitt MA: Tylavsky FA: Cross-sectional associations between trunk muscle composition: back pain: and physical function in the health: aging and body composition study. The journals of gerontology Series A: Biological sciences and medical sciences 2005: 60(7):882-887.

38. Pang W: Lou N: Jin C: Hu C: Arvine C: Zhu G: Shen X: Combination of preoperative platelet/lymphocyte and neutrophil/lymphocyte rates and tumor-related factors to predict lymph node metastasis in patients with gastric cancer. European journal of gastroenterology \& hepatology 2016: 28(5):493-502.

39. Porrata LF: Ristow K: Habermann TM: Ozsan N: Dogan A: Macon W: Colgan JP: Witzig TE: Inwards DJ: Ansell SM et al: Absolute monocyte/lymphocyte count prognostic score is independent of immunohistochemically determined cell of origin in predicting survival in diffuse large B-cell lymphoma. Leukemia \& lymphoma 2012: 53(11):2159-2165.

40. Onodera T: Goseki N: Kosaki G: [Prognostic nutritional index in gastrointestinal surgery of malnourished cancer patients]. Nihon Geka Gakkai zasshi 1984: 85(9):1001-1005.

41. Gomez-Perez SL: Haus JM: Sheean P: Patel B: Mar W: Chaudhry V: McKeever L: Braunschweig C: Measuring Abdominal Circumference and Skeletal Muscle From a Single Cross-Sectional Computed Tomography Image: A Step-by-Step Guide for Clinicians Using National Institutes of Health ImageJ. JPEN J Parenter Enteral Nutr 2016: 40(3):308-318.

42. Baumgartner RN: Koehler KM: Gallagher D: Romero L: Heymsfield SB: Ross RR: Garry PJ: Lindeman RD: Epidemiology of sarcopenia among the elderly in New Mexico. American journal of epidemiology 1998: 147(8):755-763.

43. Yoshizumi T: Shirabe K: Nakagawara H: Ikegami T: Harimoto N: Toshima T: Yamashita Y: Ikeda T: Soejima Y: Maehara Y: Skeletal muscle area correlates with body surface area in healthy adults. Hepatol Res 2014: 44(3):313-318. 
44. Wan Q: Lin C: Li X: Zeng W: Ma C: MRI assessment of paraspinal muscles in patients with acute and chronic unilateral low back pain. Br J Radiol 2015: 88(1053):20140546.

45. Abe H: Takei K: Uematsu T: Tokura Y: Suzuki I: Sakamoto K: Nishihara D: Yamaguchi Y: Mizuno T: Nukui A et al: Significance of sarcopenia as a prognostic factor for metastatic urothelial carcinoma patients treated with systemic chemotherapy. International journal of clinical oncology 2018 : 23(2):338-346.

46. Wronska A: Kmiec Z: Structural and biochemical characteristics of various white adipose tissue depots. Acta physiologica (Oxford: England) 2012: 205(2):194-208.

47. Lou N: Chi CH: Chen XD: Zhou CJ: Wang SL: Zhuang CL: Shen X: Sarcopenia in overweight and obese patients is a predictive factor for postoperative complication in gastric cancer: A prospective study. European journal of surgical oncology : the journal of the European Society of Surgical Oncology and the British Association of Surgical Oncology 2017: 43(1):188-195.

48. Cologne J: Hsu WL: Abbott RD: Ohishi W: Grant EJ: Fujiwara S: Cullings HM: Proportional hazards regression in epidemiologic follow-up studies: an intuitive consideration of primary time scale. Epidemiology (Cambridge: Mass) 2012: 23(4):565-573.

49. Bozzetti F: Marubini E: Bonfanti G: Miceli R: Piano C: Gennari L: Subtotal versus total gastrectomy for gastric cancer: five-year survival rates in a multicenter randomized Italian trial. Italian Gastrointestinal Tumor Study Group. Annals of surgery 1999: 230(2):170-178.

50. Korean Practice Guideline for Gastric Cancer 2018: an Evidence-based: Multi-disciplinary Approach. Journal of gastric cancer 2019: 19(1):1-48.

51. Eo WK: Chang HJ: Suh J: Ahn J: Shin J: Hur JY: Kim GY: Lee S: Park S: Lee S: The Prognostic Nutritional Index Predicts Survival and Identifies Aggressiveness of Gastric Cancer. Nutrition and cancer 2015: 67(8):1260-1267.

52. Ishizuka M: Oyama Y: Abe A: Tago K: Tanaka G: Kubota K: Prognostic nutritional index is associated with survival after total gastrectomy for patients with gastric cancer. Anticancer research 2014: 34(8):4223-4229.

53. Watanabe M: Iwatsuki M: Iwagami S: Ishimoto T: Baba Y: Baba H: Prognostic nutritional index predicts outcomes of gastrectomy in the elderly. World journal of surgery 2012: 36(7):1632-1639.

\section{Figures}




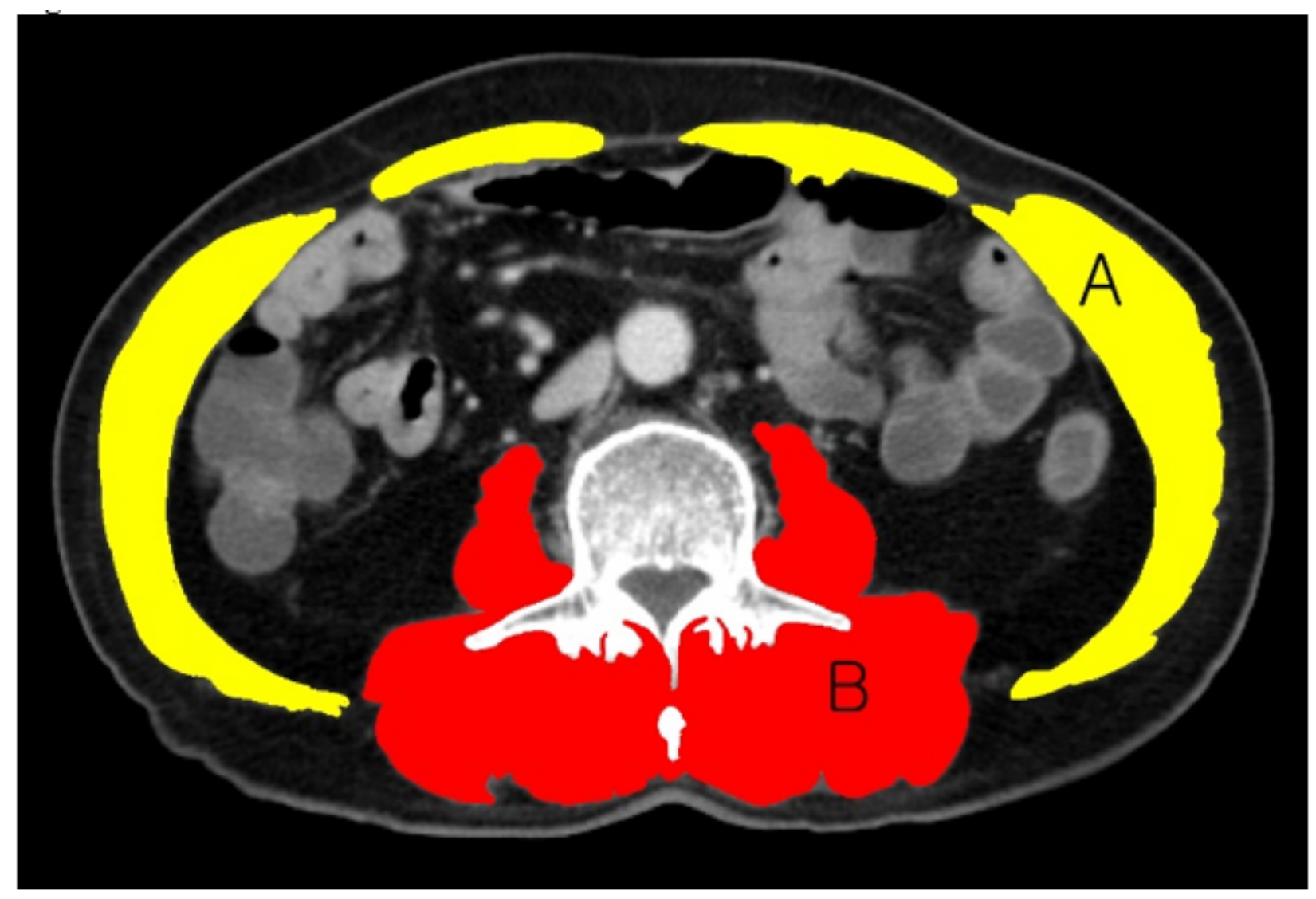

\section{Figure 1}

The cross-sectional area at the third lumbar vertebra level. The group of anterolateral abdominal wall musculature (i.e.: transversus abdominus: external and internal obliques: and rectus abdominus) (A) and the group of paraspinal muscles (i.e.: erector spinae: multifidus: quadratus lumborum and psoas) (B) are shown. The SMI and SMMA are measured in areas (A) and (B): while the PMI and PMMA are measured in area (B). SMI: skeletal muscle index; SMMA: mean attenuation within total abdominal wall musculature; PMI: paraspinal muscle index; PMMA: mean attenuation within paraspinal muscle 
(A)

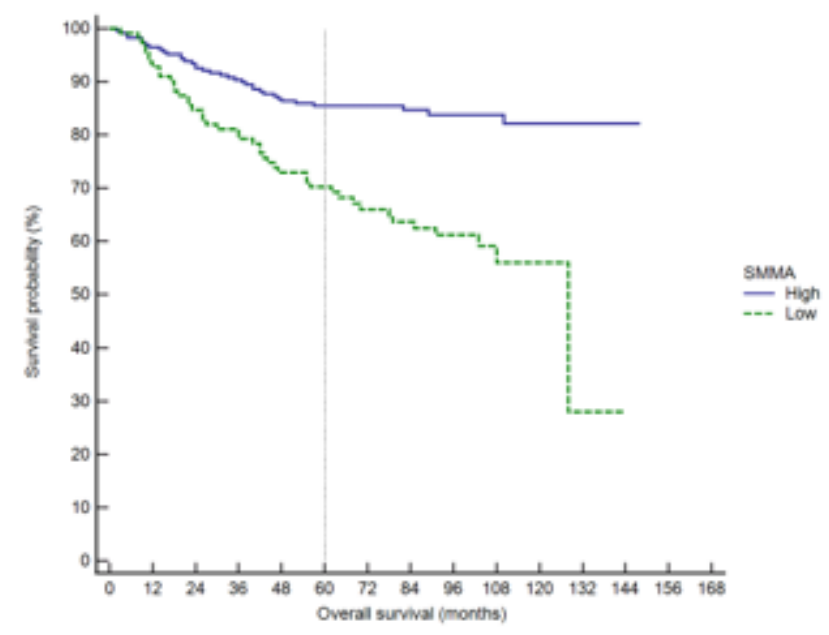

(C)

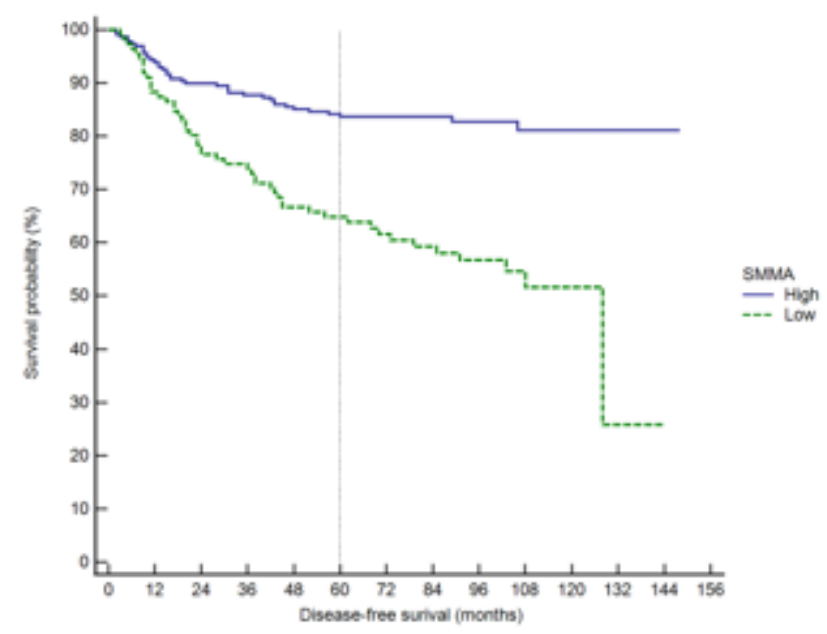

(B)

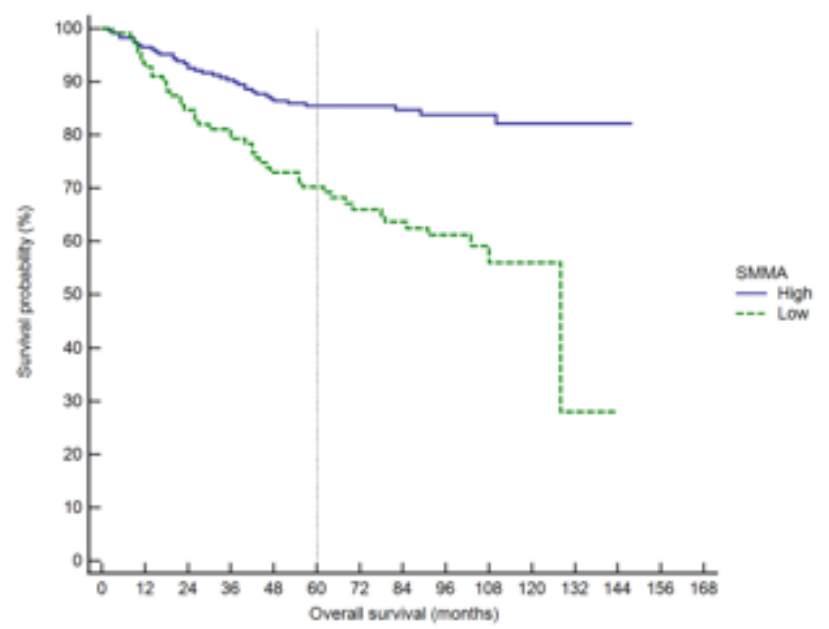

(D)

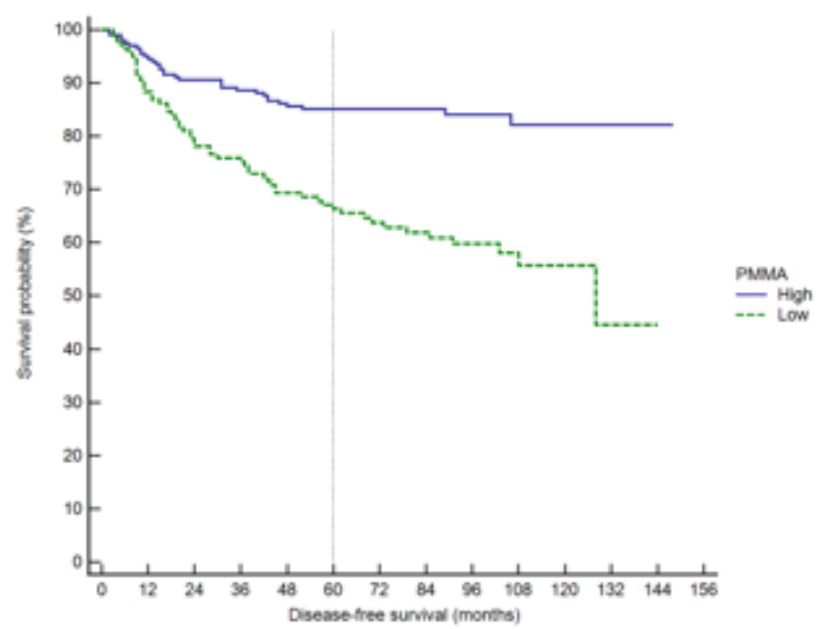

Figure 2

The overall survival curves according to SMMA (A) and PMMA (B): and the disease-free survival curves according to SMMA (C) and PMMA (D). SMMA: mean attenuation within total abdominal wall musculature; PMMA: mean attenuation within paraspinal muscle 